\title{
Einleitung
}

\section{Die Handschrift}

A >Ambraser Heldenbuch`, Wien, Österreichische $\mathrm{Na}^{-}$ tionalbibliothek, Cod. ser. nova 2663.

Material: Pergament; Vorsatzblätter aus Papier; Einband aus Pappe mit Kalbslederüberzug unter Verwertung von Teilen des ursprünglichen Ledereinbands.

Umfang: $\quad 5+238$ Blätter.

Maße: $\quad$ Blätter: $460 \mathrm{~mm} \times 360 \mathrm{~mm}$; Schriftspiegel: $360 \mathrm{~mm} \times 235 \mathrm{~mm} ; 3$ Spalten zu $66-$ 69 Zeilen.

Foliierung: Lateinisch auf den Recto-Seiten (fol. I28 fehlt, fol. I53 ist zweimal gezählt); arabisch in Blei auf den Verso-Seiten (aus dem 20. Jahrhundert).

Inhalt:

$I^{*} r-4^{*} \mathrm{v}: \quad$ Tabula

$5^{*} \mathrm{v}: \quad$ Frontispiz

Ira-2rb: Der Stricker: >Die Frauenehre< (unvollständig) (Handschrift d)

2va-5vc: $\quad>$ Mauritius von Craûn (Unikat)

5vc-22rc: Hartmann von Aue: >Iwein< (Handschrift d)

22rc-26va: Hartmann von Aue: `Die Klager (auch

>Erstes Büchlein`) (Unikat)

26va-28rb: >Das Büchlein (auch `Zweites Büchlein`) (Unikat)

28rb-3orb: >Der Mantek (Unikat)

3orb-5ovb: Hartmann von Aue: >Erec (Unikat)

5ovc-75ra: >Dietrichs Flucht< (Handschrift d)

75rb-92rb: >Rabenschlacht< (Handschrift d)

95ra-I27va: >Nibelungenlied (Handschrift d)

I3IVa-I39vb: >Nibelungenklager (Handschrift d)

I40ra-I66ra: >Kudrun (Unikat)

I66rb-195vc: >Biterolf und Dietleib< (Unikat)
I96ra-205vb: >Ortnit< (Handschrift A)

205vb-2I4vc: ,Wolfdietrich A (Unikat)

215ra-216vb: >Die böse Frau< (Unikat)

217ra-217va: Herrand von Wildonie: ১Die treue Gattin (Unikat)

217vb-2I8rc: Herrand von Wildonie: >Der betrogene

Gatter (Unikat)

2I8rc-2I9vc: Herrand von Wildonie: >Der nackte Kaiser (Unikat)

219vc-220va: Herrand von Wildonie: >Die Katze (Unikat)

220va-225rb: Ulrich von Liechtenstein: `Frauenbuch (Unikat)

225rb-229rb: Wernher der Gärtner: `Helmbrechtく (Handschrift A)

229rb-233vb: Der Stricker: >Pfaffe Amis< (Handschrift W)

234ra-235rb: Wolfram von Eschenbach: >Titurek (Handschrift $\mathrm{H}$ )

235va-237vc: >Brief des Priesterkönigs Johannes` (Unikat)

Zu Beginn des I6. Jahrhunderts beauftragte Maximilian I. den Bozner Zöllner Hans Ried mit der Niederschrift einer Sammelhandschrift, die später als >Ambraser Heldenbuch $<$ in die Literarturgeschichte einging. ${ }^{.}$Alle Texte dieses Prachtkodex wurden in einer Hand von Hans Ried im Zeitraum zwischen 1504 und 1516 niedergeschrieben und weisen trotz des beachtlichen Umfangs von $5+238$ Pergamentblättern hinsichtlich Qualität und Erscheinungsbild größte Konsistenz auf. ${ }^{2}$ Bemerkenswert an dieser prunkvollen Sammelhandschrift ist aber vor allem die Tatsache, dass von ihren 25 Werken 15 im >Ambraser Heldenbuch unikal überliefert sind. Hierzu zählen so wichtige Texte des mittelhochdeutschen Literaturkanons wie `Erec<, >Kudrun oder `Mauritius von Craûn<. Auch die weitere Manuskriptlage zum Schreiber Hans Ried ist ungewöhnlich, da neben dem >Ambraser Heldenbuch auch relativ viele Urkunden und Gebrauchstexte aus seiner Feder überliefert sind. ${ }^{3}$

I Zur Beauftragung des Schreibers siehe Alisade (2019). Zu aktuellen Themen und Forschungsarbeiten zum >Ambraser Heldenbuch siehe Klarer (2019).

2 Zur kodikologischen Beschreibung des >Ambraser Heldenbuchs` siehe Menhardt (I96r: S. I469-I478), Bäuml (I969a), Unterkircher (1973), Janota (1978), Gärtner (2015) und Tratter (2019).

3 Zu den Autographen von Hans Ried siehe Mura (2007) und Alisade (2019). 


\section{Wissenschaftliche Relevanz einer Gesamttranskription des $>$ Ambraser Heldenbuchs}

Das >Ambraser Heldenbuch liegt bereits seit geraumer Zeit als Faksimile (1973) vor und auch alle Texte des A Ambraser Heldenbuchs sind über gedruckte Editionen (meist in normalisierter Form als Rückübertragungen in standardisiertes Mittelhochdeutsch) zugänglich. Jedoch gibt es neben vereinzelten Transkriptionen ausgewählter Werke bisher keine umfassende zeichengetreue Gesamttranskription des Kodex. Dies ist umso verwunderlicher, da sich seit vielen Jahrzehnten Stimmen mehren, die einer Gesamtbeurteilung der Sprache und Gesamttranskription des >Ambraser Heldenbuchs höchste Priorität zusprechen (z. B. Leitzmann 1935; Gärtner 2006; Mura 2007). Hierbei werden vielfältige, vor allem editionsphilologische Gründe für eine zeichengetreue Transkription des >Ambraser Heldenbuchs als »dringendes Forschungsdesiderat « (Homeyer/Knor 20I5: S. 98) ins Feld geführt. Um diese Forschungslücke zu schließen, arbeitet die vorliegende Gesamtausgabe mit zwei korrespondierenden Transkriptionen. Die allographische Transkription differenziert neben den Graphemen die allographischen Varianten, die in der Handschrift vorkommen. Dabei werden ausgehend von der Federführung allographische Varianten von Graphemen isoliert, klassifiziert und in der Transkription verwendet. Parallel dazu werden die allographischen Varianten in der diplomatischen Transkription, die auf der allographischen Transkription beruht, vereinheitlicht.

Eine so gestaltete Gesamttranskription des >Ambraser Heldenbuchs s ist aus folgenden Gründen von Relevanz:

- Eine der umfangreichsten Sammelhandschriften mittelhochdeutscher Literatur:

Das `Ambraser Heldenbuch ist mit 25 wichtigen mittelalterlichen literarischen Erzähltexten, von denen is im >Ambraser Heldenbuch umfangreichste Kodex (ca. 600 ooo Wörter) seiner Art.

- Korpus in einer Schreiberhand:

Von einem einzelnen Schreiber niedergeschrieben bietet das >Ambraser Heldenbuch (zusammen mit den Urkunden Hans Rieds) eine exzellente Materialbasis für ein allographisches Korpus unter Berücksichtigung graphemischer Varianten, Superskripta, Abkürzungs- und Interpunktionszeichen der riedschen Schreiberhand.

- Literarische Sprache:

Obwohl die Texte im >Ambraser Heldenbuch I2. und I3. Jahrhundert stammen, sind sie dort aus- schließlich in frühneuhochdeutscher Sprache festgehalten. Im >Ambraser Heldenbuch manifestiert sich eine literarische Sprache, die sich von anderen überlieferten Autographen Hans Rieds (aus einem dezidiert nichtliterarischen Kontext) abhebt. Bereits 1935 beklagte Albert Leitzmann das Fehlen einer »zusammenfassende[n] untersuchung über die copistenleistung Johannes Rieds und damit eine $[\mathrm{r}]$ gesamtbeurteilung ihres sprachlichen charakters in all seinen einzelheiten« (S. I89). 1969 spezifizierte Franz H. Bäuml: »Bei einer Behandlung der Sprache der Handschrift ist äußerste Vorsicht geboten. Es ist erstens zu bedenken, daß die Handschrift eine Abschrift einer spätmhd. oder ffnhd. Vorlage ist, über deren sprachliche Beschaffenheit wir so gut wie nichts wissen. Was die sprachlichen Eigenschaften der Vorlage anbelangt, ist es ganz unmöglich, auf Grund des vorliegenden Materials zu irgendwelchen auch nur halbwegs sicheren Schlüssen zu kommen. Und es muß zweitens die Möglichkeit angenommen werden, daß diese uns in so großem Maße unbekannte Vorlage in sprachlicher Hinsicht den Schreiber Hans Ried in Einzelheiten beeinflußt hat. Einerseits kann er also von seiner Vorlage den Einflüssen seines eigenen Dialektes teilweise entzogen worden sein, anderseits kann er sich fern genug von dialektischen Einschlägen gehalten haben, so daß eine Untersuchung, den Dialekt Hans Rieds festzustellen, auf äußerst unsicherem Boden fußen würde.« (Bäuml r969b: S. 32)

- Unikale Überlieferung:

Der Großteil der bisherigen Editionen von unikalen Werken des >Ambraser Heldenbuchs` versucht, eine Rücktransposition in normalisiertes Mittelhochdeutsch zu vollführen. Hierfür betont Kurt Gärtner in der Einleitung seiner 7. Auflage des $>$ Erec $<$ die Nützlichkeit einer »vollständigen Transkription" (2006: S. XX) des >Ambraser Heldenbuchs $\triangleleft$ Es gibt folglich bisher keine vollständige Gesamtausgabe aller Texte des >Ambraser Heldenbuchs als zeichengetreue Transkription ohne den Versuch einer Normalisierung oder Standardisierung. Gerade für die im >Ambraser Heldenbuch überlieferten Texte (wie z. B. Hartmanns >Erec ) sehen Susanne Homeyer und Inta Knor (2015) das große Potential einer Gesamttranskription: »[F]ehlt doch die Gesamtschau auf den Schreibusus Rieds im Rahmen seiner Abschrift des >Ambraser Heldenbuches`, um mögliche Vorlagenreflexe von Texteingriffen, Wortschatzwandel oder individuellen Schreibgewohnheiten zu trennen.«(S. 98) Die vorliegende Gesamttranskription sollte damit anschlussfähig für unterschiedliche 
Editionsbemühungen von Einzeltexten des >Ambraser Heldenbuchs« sein.

Zusammenfassend liegt der unmittelbare Grund für eine Gesamttranskription des >Ambraser Heldenbuchs` darin, dass 15 der 25 Texte der Sammelhandschrift unikal überliefert sind. Die Unikate sind die einzige Quelle für die Erarbeitung von Editionen dieser Werke. Auf der Basis der vorliegenden Gesamttranskription ließe sich die »Schreibsprache Rieds« (Gärtner 2006: S. XX), die er für die Niederschrift seiner Texte im >Ambraser Heldenbuch verwendet hat, besser greifbar machen. Im Umkehrschluss können diese Erkenntnisse Rückschlüsse auf seine (nichtüberlieferten) Vorlagen ermöglichen und damit Editionen der unikal überlieferten Werke potentiell verfeinern und schärfen. Darüber hinaus lässt eine Gesamttranskription eine Vielzahl von Untersuchungen zu und legt damit den Grundstein für zukünftige Forschungsprojekte in unterschiedlichen Disziplinen rund um das >Ambraser Heldenbuch

Im Rahmen des ÖAW-go!digital-2.o-Forschungsprojekts »Ambraser Heldenbuch: Transkription und wissenschaftliches Datenset « (Projektleitung Mario Klarer) wurde erstmals das gesamte >Ambraser Heldenbuch (allographisch) transkribiert und als digitales Datenset vorgelegt. Die gedruckte Gesamtausgabe basiert auf diesem Datenset und wurde mit großzügiger Unterstützung durch das Amt der Tiroler Landesregierung und die Landeshauptstadt Innsbruck im Rahmen des Projekts »Kaiser Maximilian goes digital: Vom `Gedächtnis` zum Datenspeicher« (Projektleitung Mario Klarer) ermöglicht.

Die vorliegende Gesamttranskription des >Ambraser Heldenbuchs versteht sich dezidiert nicht als kritische Edition und will auch in keiner Weise mit bereits existierenden kritischen Editionen einzelner Texte der Sammelhandschrift in Konkurrenz treten. Wichtigstes Ziel dieser Gesamttranskription ist es, erstmals alle Texte des >Ambraser Heldenbuchs` als Gegenüberstellung von $\mathrm{Ma}^{-}$ nuskriptbild, allographischer Transkription und diplomatischer Transkription zugänglich zu machen:

\section{Manuskriptbild in Originalgröße}

Der Scan des entsprechenden Manuskriptausschnitts erlaubt einen direkten Vergleich mit den zwei parallel dazu angeordneten Transkriptionen. Zusätzlich werden die Buchmalereien abgebildet, die sich am Seitenrand links oder rechts der Textspalten befinden. ${ }^{4}$

\section{Allographische Transkription gemäß dem Zeilenumbruch des Manuskripts}

In der allographischen Transkription werden die allographischen Varianten der riedschen Schreiberhand abgebildet, wobei auf Standardisierungen verzichtet wird. Zudem wird dem Zeilenumbruch des Manuskripts gefolgt, sodass eine synoptische Gegenüberstellung des Manuskripts und der allographischen Transkription ermöglicht wird.

\section{Diplomatische Transkription gemäß Versen und Strophen sowie deren Nummerierung}

Eine dazu parallel angeordnete diplomatische Transkription vereinheitlicht gegenüber der allographischen Transkription allographische Varianten sowie Superskripta und löst Abbreviaturen auf, um die Transkription allgemein zitierfähig zu machen. Die diplomatische Transkription ist nach Versen und, so diese vorhanden, Strophen umbrochen, welche gemäß etablierten Editionen der Texte (siehe 4.I. Konsultierte Editionen für Nummerierung der Verse und Strophen) nummeriert sind. Damit wird ein Vergleich mit Editionen der einzelnen Texte ermöglicht.

Um die verschiedenen Ebenen der Transkription darzustellen, sind in der vorliegenden Druckausgabe neben dem Bild des Manuskripts die allographische Transkription und eine zitierfähige, vereinfachte Transkription parallel angeordnet.

\section{Einrichtung der Ausgabe}

\section{I. Manuskriptbild (linke Seite der Gesamtausgabe)}

Das >Ambraser Heldenbuch $<$ besteht aus $5+238$ Pergamentblättern $(460 \mathrm{~mm} \times 360 \mathrm{~mm})$, die mit Ausnahme des Inhaltsverzeichnisses den Text in drei Spalten wiedergeben. Um die Manuskriptbilder in Originalgröße wiedergeben zu können, wird in der Gesamtausgabe pro Seite jeweils eine halbe Spalte aus dem >Ambraser Heldenbuch abgebildet $(220,13 \mathrm{~mm} \times 105,83 \mathrm{~mm}$ oder $220,13 \mathrm{~mm} \times$ I60,87 mm). Die Zeilennummerierung steht links oder, wenn sich links des Texts Buchmalereien befinden, rechts neben dem Manuskriptbild. Aufgrund der Wölbung der Pergamentseiten und der Linienführung Hans Rieds kann es zu leichten Diskrepanzen zwischen der tatsächlichen Position einer Zeile im Manuskriptbild und der Zeilennummerierung kommen.

4 Zum Buchschmuck des >Ambraser Heldenbuchs« siehe Domanski (2019). 


\subsection{Allographische Transkription (rechte Seite der Gesamtausgabe, linke Spalte)}

Auf der rechten Seite der Gesamtausgabe sind zwei getrennte Transkriptionen jenes Textes wiedergegeben, dessen Scan auf der linken Seite der Gesamtausgabe abgebildet ist. Die links angeordnete Transkription gibt den Manuskriptscan einer halben Spalte allographisch wieder: Hierzu zählen die Beibehaltung des Zeilenumbruchs des Manuskripts (bei dem die Versenden nicht mit den Zeilenenden zusammenfallen) und die allographische Wiedergabe der Buchstaben, Superskripta, Interpunktionszeichen sowie der Abbreviaturen gemäß den Transkriptionsrichtlinien (siehe Transkriptionszeichen der allographischen Transkription). So werden beispielsweise die Allographe $\langle S\rangle,\langle\beta\rangle,\langle\sigma\rangle,\langle\delta\rangle$ und $\langle\delta$ für das Graphem $\langle\mathrm{S}\rangle$ in der allographischen Transkription verwendet. Ebenso werden ausgepunktete oder durchgestrichene Textpassagen in der allographischen Transkription mittels Durchstreichung wiedergegeben. Nicht wiedergegeben wird die exakte Größe von Lombarden, wobei jedoch deren Farbe (rot oder blau) als Orientierungshilfe beibehalten wird. Die restlichen Initialen, die sich zu Beginn der einzelnen Texte oder âventiuren befinden, werden durch eine Darstellung über drei Zeilen gekennzeichnet, die jedoch nicht der tatsächlichen Größe im Manuskript entspricht. Incipits und Explicits werden in der Transkription wie im Manuskript farblich (rot) hervorgehoben. Auf die Kennzeichnung der Rubrizierungen von Majuskeln wird zur Gänze verzichtet, die aber über den Manuskriptscan leicht erschließbar sind.

Um die Benutzung und den Vergleich von Manuskriptscan und linker Transkriptionsspalte möglichst effizient zu gestalten, wird eine synoptische Darstellung gewählt. Aufgrund der Wölbung des Pergaments und der Linienführung Hans Rieds kann es jedoch auch hier vereinzelt zu leichten Positionsabweichungen zwischen den Zeilen im Scan und der allographischen Transkription kommen.

\section{Richtlinien für die allographische Transkription}

Im Folgenden soll ein kurzer Überblick über die von Hans Ried im >Ambraser Heldenbuch ` verwendeten Buchstaben, Superskripta, Abkürzungs- und Interpunktionszeichen gegeben werden. Bei der Charakterisierung und Identi- fizierung der Zeichen für die allographische Transkription wurde als Kriterium vor allem die Federführung Hans Rieds herangezogen. Trotz der großen Einheitlichkeit der Schreiberhand und dem Anspruch der vorliegenden allographischen Transkription musste in wenigen Fällen eine normative Auswahl getroffen werden. Hierzu zählen:

- Großbuchstaben-Varianten:

Insbesondere bei den Großbuchstaben hat Hans Ried einen relativ großen Facettenreichtum praktiziert. Da die ohnedies spärlich verwendeten Großbuchstaben es kaum erlauben, eigenständige Varianten klar voneinander abzugrenzen, wird in der vorliegenden Ausgabe bewusst auf Differenzierungen der Großbuchstabenvarianten verzichtet.

- Superskripta:

Die größte Herausforderung für die allographische Transkription des >Ambraser Heldenbuchs stellen die von Hans Ried verwendeten Superskripta dar. In der vorliegenden Transkription wird prinzipiell zwischen vier verschiedenen Superskripta unterschieden. Jedoch lassen diese Superskripta - mehr als alle anderen verwendeten Zeichen Hans Rieds - besonders fließende Übergänge in der Ausführung erkennen. In vielen Fällen ist daher eine eindeutige Zuordnung rein aufgrund der Linienführung Hans Rieds nicht möglich. Zur Unterscheidung wurden neben der Federführung Hans Rieds für jeden Einzelfall kontext- und wortspezifische Kriterien (z. B. Differenzierung ähnlich aussehender Grapheme wie 〈 $\mathrm{u}$ ) und 〈n〉 oder Kennzeichnung von Umlauten und Diphthongen) herangezogen.

\section{Transkriptionszeichen der allographischen Transkription}

Im Folgenden werden die im >Ambraser Heldenbuch verwendeten Zeichen dokumentiert und den Transkriptionszeichen der vorliegenden Gesamtausgabe gegenübergestellt. Die Abbildungen der von Hans Ried verwendeten Zeichen sind dabei gegenüber dem Original um den Faktor 2 vergrößert. Bei den allographischen Varianten ist jeweils als Zusatzinformation angegeben, Allographe welchen Graphems sie sind. Zu diesen Graphemen werden die allographischen Varianten in der diplomatischen Transkription vereinheitlicht. 


\section{Kleinbuchstaben}

\begin{tabular}{|c|c|c|c|}
\hline $\begin{array}{c}\text { Transkriptions- } \\
\text { zeichen }\end{array}$ & Name & Unicode & Abbildung \\
\hline $\mathfrak{d}$ & Latin Small Letter A & $\mathrm{U}+006 \mathrm{I}$ & fol. XCVra 1.2 \\
\hline & Latin Small Letter B & $\mathrm{U}+0062$ & 6 \\
\hline & Latin Small Letter C & $\mathrm{U}+0063$ & fol. XCVrb l. $7 \mathrm{ab}$ imo \\
\hline & Latin Small Letter D & $\mathrm{U}+0064$ & fol. XCVrb 1. $5 \mathrm{ab}$ imo \\
\hline & Partial Differential & $\mathrm{U}+2202$ & $\begin{array}{l}8 \\
\text { fol. XXXVrc 1. i6 ab imo }\end{array}$ \\
\hline \multicolumn{4}{|c|}{ Variante des Kleinbuchstabens $\langle\mathrm{d}\rangle$} \\
\hline e & Latin Small Letter E & $\mathrm{U}+0065$ & fol. XCVrc 1.8 \\
\hline$f$ & Latin Small Letter F & U+oo66 & $\int_{\text {fol. XCVrb } 1.5 \mathrm{ab} \text { imo }}$ \\
\hline$\delta$ & Latin Small Letter G & $\mathrm{U}+0067$ & fol. XCVra $1.2 \mathrm{I}$ \\
\hline
\end{tabular}




\begin{tabular}{|c|l|l|l|}
\hline $\mathbf{h}$ & Latin Small Letter $\mathrm{H}$ & U+oo68 & \\
\hline $\mathbf{6}$ & $\begin{array}{l}\text { Latin Small Letter Heng } \\
\text { with Hook }\end{array}$ & U+o267 & fol. XCVrc 1.28 \\
\hline
\end{tabular}

Variante des Kleinbuchstabens « $h$ », die vor allem bei Abkürzungen und an Zeilenenden auftritt

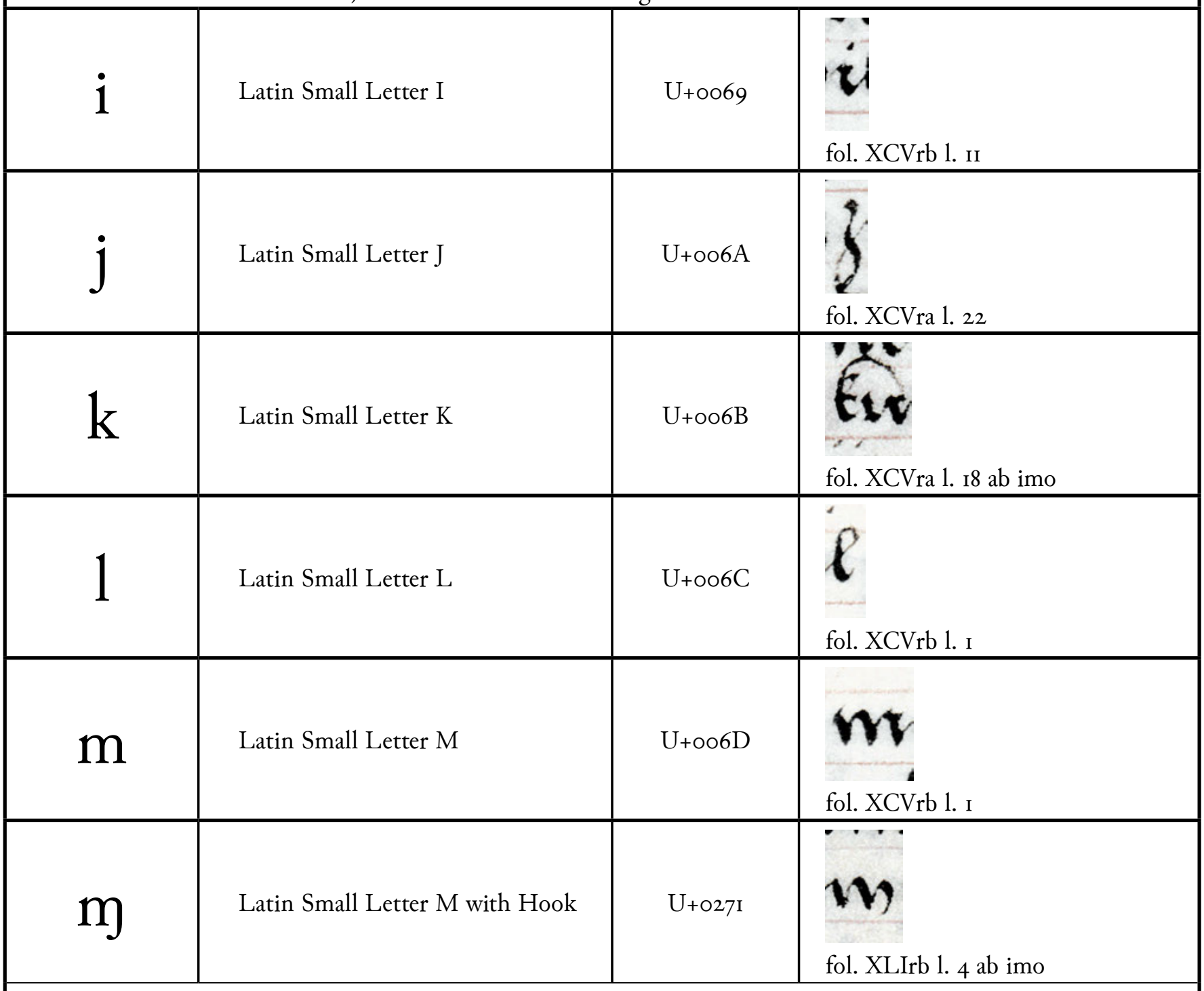

Variante des Kleinbuchstabens 〈m〉, die am Ende von Wörtern auftritt, jedoch nicht bei allen Wörtern, die mit 〈m〉 enden 


\begin{tabular}{|c|c|c|c|}
\hline$n$ & Latin Small Letter N & $\mathrm{U}+006 \mathrm{E}$ & $\begin{array}{l}\text { ft } \\
\text { fol. XCVrc l. }\end{array}$ \\
\hline$\eta$ & Latin Small Letter Eng & $\mathrm{U}+\mathrm{OI}_{4} \mathrm{~B}$ & $\begin{array}{l}\text { (1) } \\
\text { fol. XCVra } 1.2\end{array}$ \\
\hline
\end{tabular}

Variante des Kleinbuchstabens «n〉, die am Ende von Wörtern auftritt, jedoch nicht bei allen Wörtern, die mit «n〉 enden

\begin{tabular}{|c|c|c|c|}
\hline 0 & Latin Small Letter O & $\mathrm{U}+006 \mathrm{~F}$ & fol. XCVra 1.8 \\
\hline & Latin Small Letter P & $\mathrm{U}+0070$ & fol. XXXVra l. i ab imo \\
\hline & Latin Small Letter Q & U+007I & fol. CLIII*va 1. II \\
\hline $\boldsymbol{r}$ & Latin Small Letter R & $\mathrm{U}+0072$ & fol. XCVra 1.2I \\
\hline 2 & Latin Small Letter R Rotunda & $\mathrm{U}+\mathrm{A} 75 \mathrm{~B}$ & fol. XCVra 1.6 \\
\hline \multicolumn{4}{|c|}{$\begin{array}{l}\text { Variante des Kleinbuchstabens }\langle\mathbf{r}>\text {, die nach Buchstaben mit Rundung nach außen auftritt, jedoch nicht nach allen } \\
\text { Buchstaben mit Rundung nach außen }\end{array}$} \\
\hline$S$ & Latin Small Letter S & $\mathrm{U}+0073$ & fol. XCVra l. $30 \mathrm{ab}$ imo \\
\hline
\end{tabular}




\begin{tabular}{|c|c|c|c|}
\hline $\mathbf{B}$ & $\begin{array}{l}\text { Latin Small Letter } \\
\text { Closed Reversed Open E }\end{array}$ & $\mathrm{U}+025 \mathrm{E}$ & 6 \\
\hline \multicolumn{4}{|c|}{ Variante des Kleinbuchstabens 〈s〉, die im Auslaut eines Wortes oder einer Silbe auftritt } \\
\hline$\sigma$ & Greek Small Letter Sigma & $\mathrm{U}+\mathrm{O}_{3} \mathrm{C}_{3}$ & fol. XVIIrc $1.5 \mathrm{ab}$ imo \\
\hline \multicolumn{4}{|c|}{ Variante des Kleinbuchstabens 〈 $\varsigma\rangle$, die im Auslaut eines Wortes oder einer Silbe auftritt } \\
\hline & Latin Small Letter Long S & $\mathrm{U}+\mathrm{OI} 7 \mathrm{~F}$ & $\int_{\text {fol. XVIIrc } 1.5 \mathrm{ab} \text { imo }}$ \\
\hline \multicolumn{4}{|c|}{ Variante des Kleinbuchstabens $\langle\mathrm{s}\rangle$, die im Anlaut oder Inlaut eines Wortes oder einer Silbe auftritt } \\
\hline & $\begin{array}{l}\text { Latin Enlarged Letter } \\
\text { Small Long S }\end{array}$ & $\mathrm{U}+\mathrm{EEDF}$ & fol. LIvc $1.2 \mathrm{ab}$ imo \\
\hline \multicolumn{4}{|c|}{ Variante des Kleinbuchstabens $\langle\mathrm{s}$, die im Anlaut oder Inlaut eines Wortes oder einer Silbe auftritt } \\
\hline & Latin Small Letter Sharp S & $\mathrm{U}+\mathrm{ooDF}$ & fol. LIIvc l. I ab imo \\
\hline$t$ & Latin Small Letter T & $\mathrm{U}+0074$ & fol. XCVrb 1. I \\
\hline ל & Latin Small Letter Tz & $\mathrm{U}+\mathrm{A} 729$ & fol. XXXVra 1.23 \\
\hline \multicolumn{4}{|c|}{ Ligatur aus den Kleinbuchstaben $\langle\mathrm{t}\rangle$ und $\langle\mathrm{z}\rangle$} \\
\hline $\mathbf{U}$ & Latin Small Letter U & $\mathrm{U}+0075$ & fol. XCVrcl. 2 \\
\hline
\end{tabular}




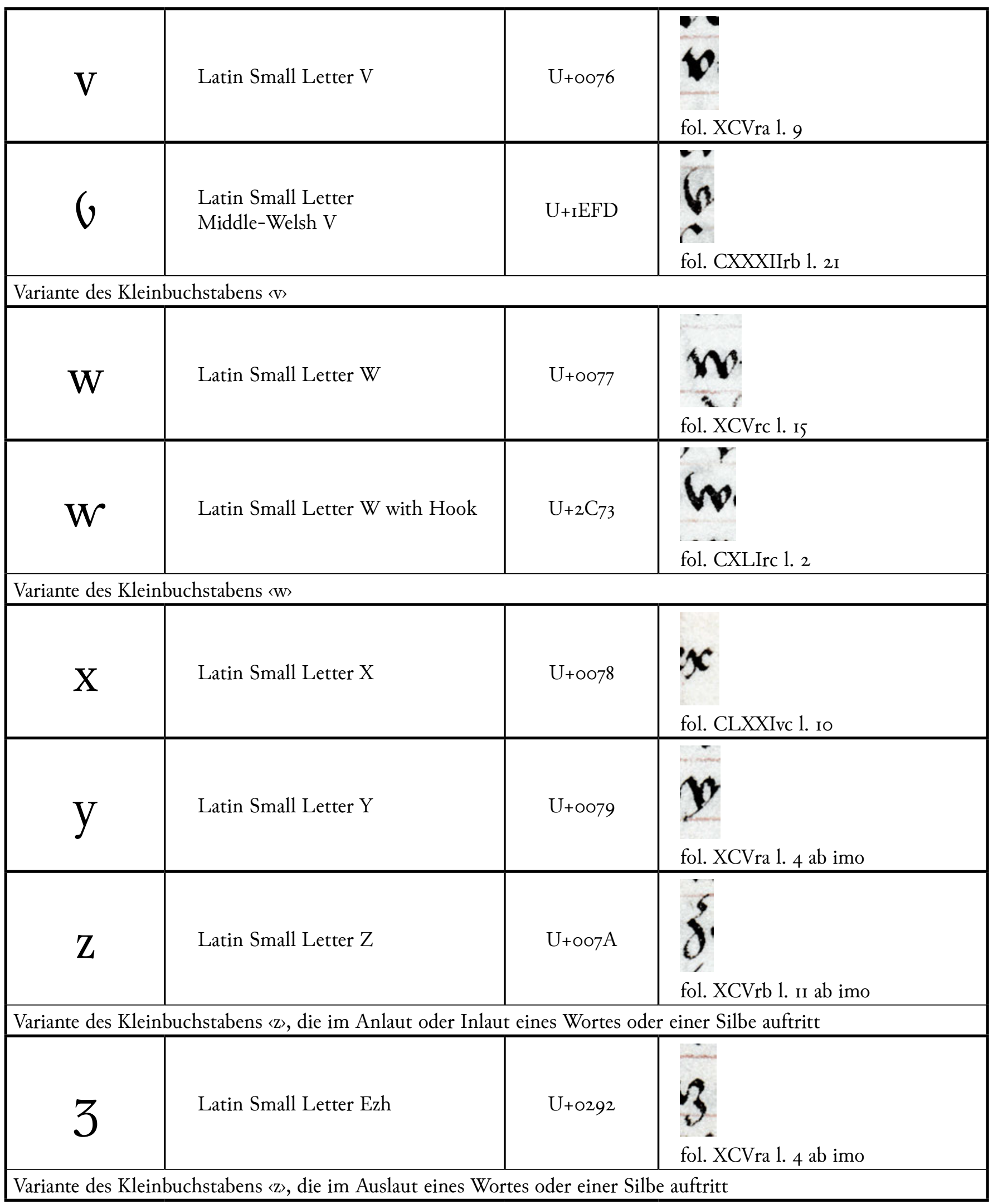




\section{Großbuchstaben}

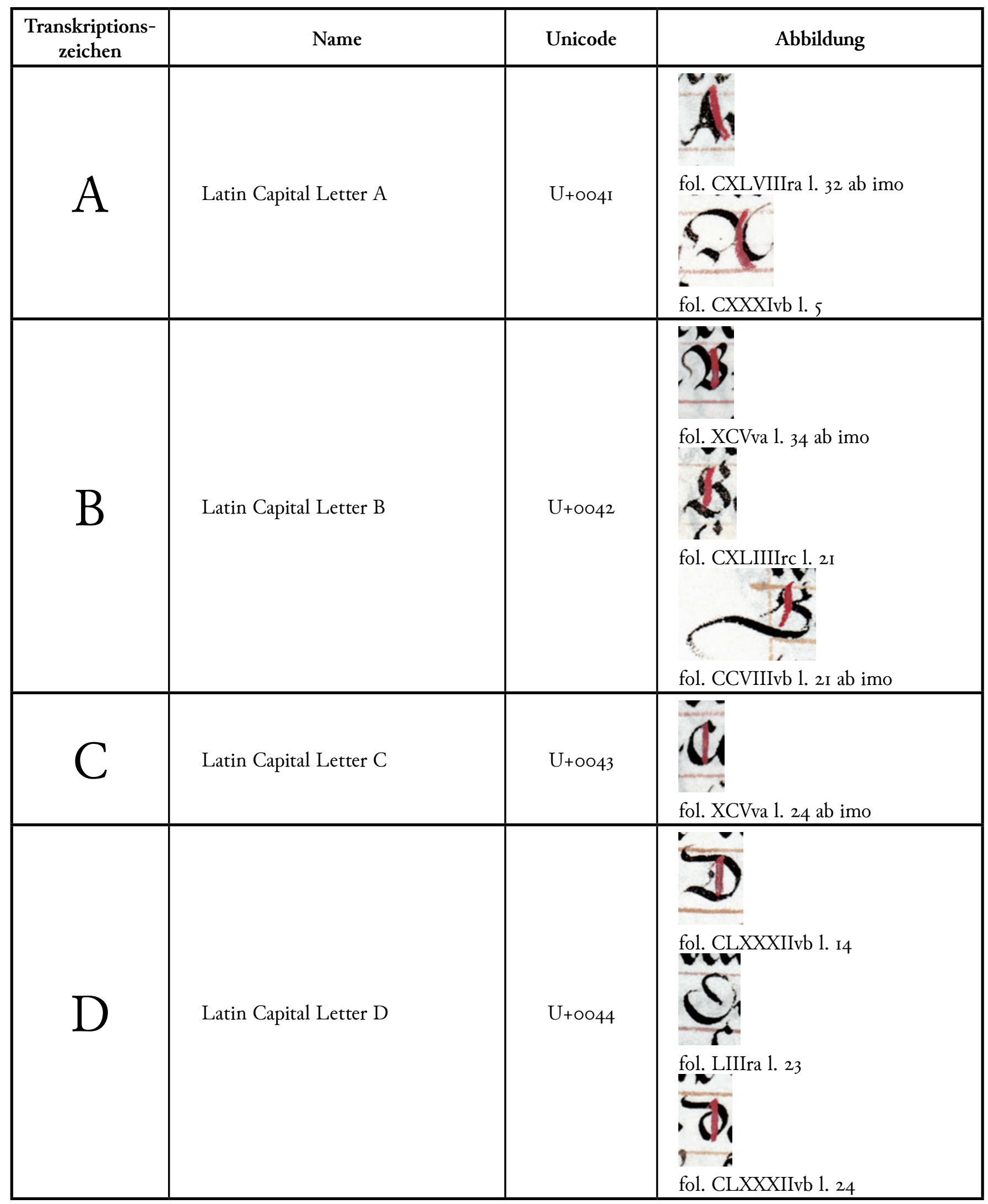




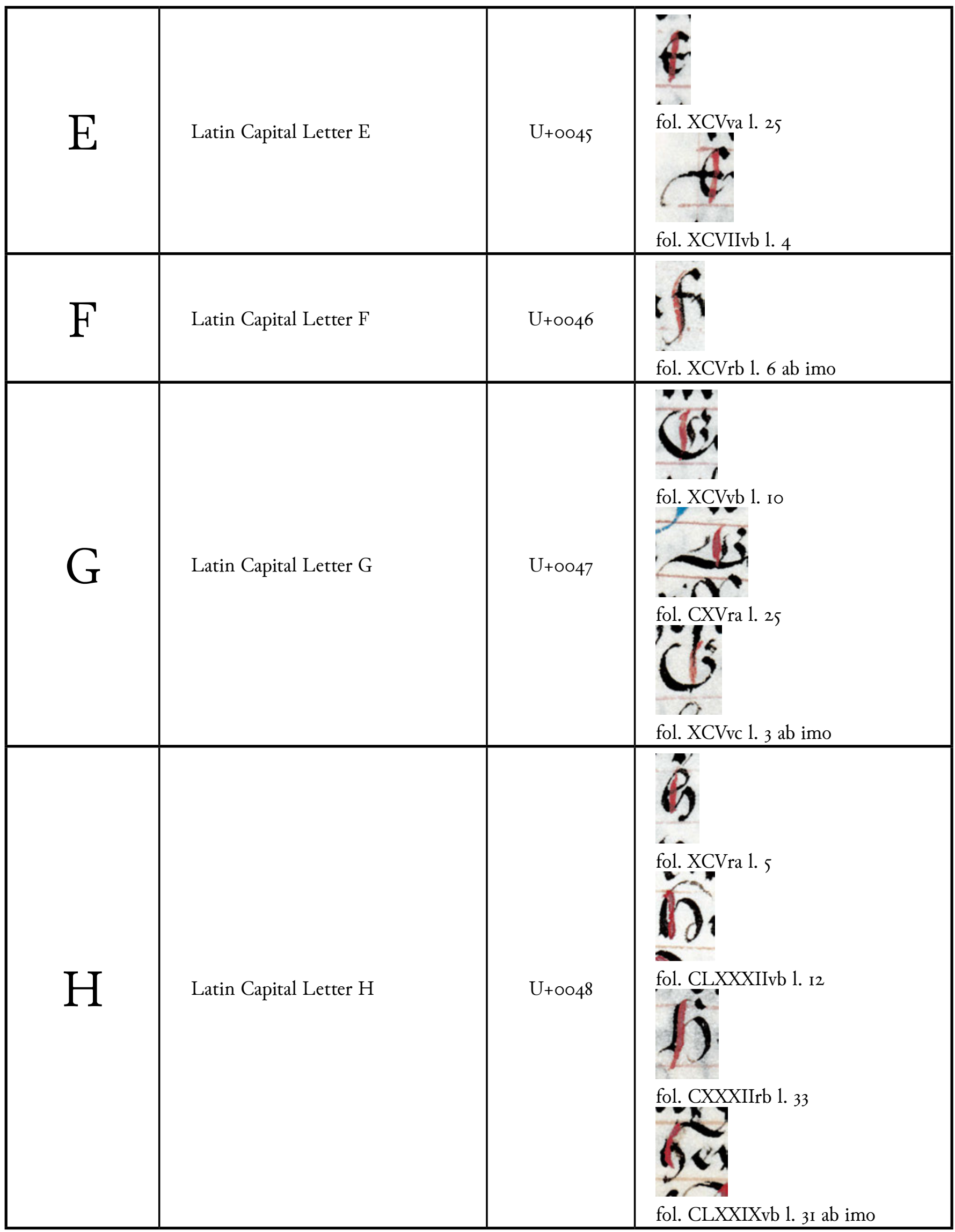




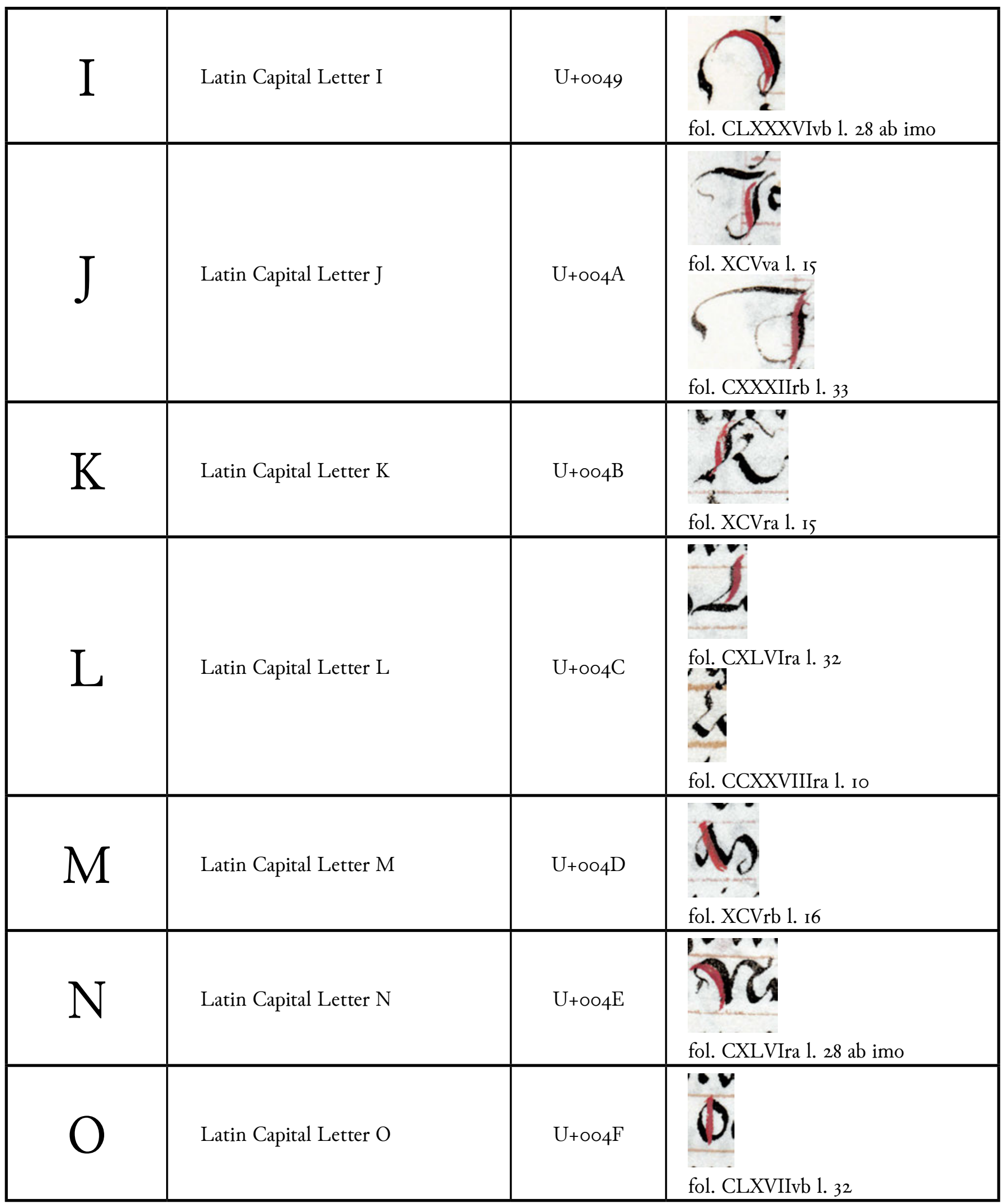




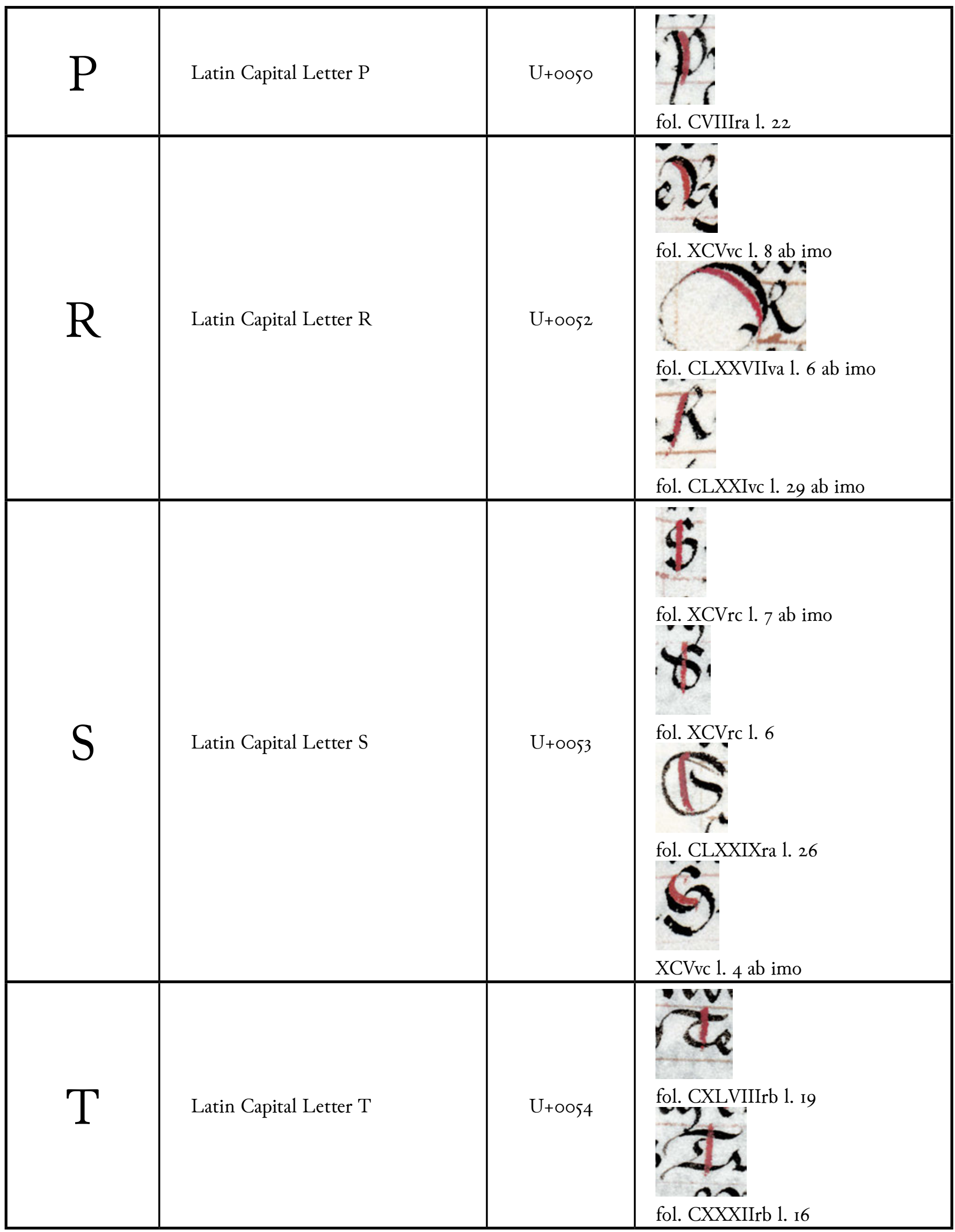




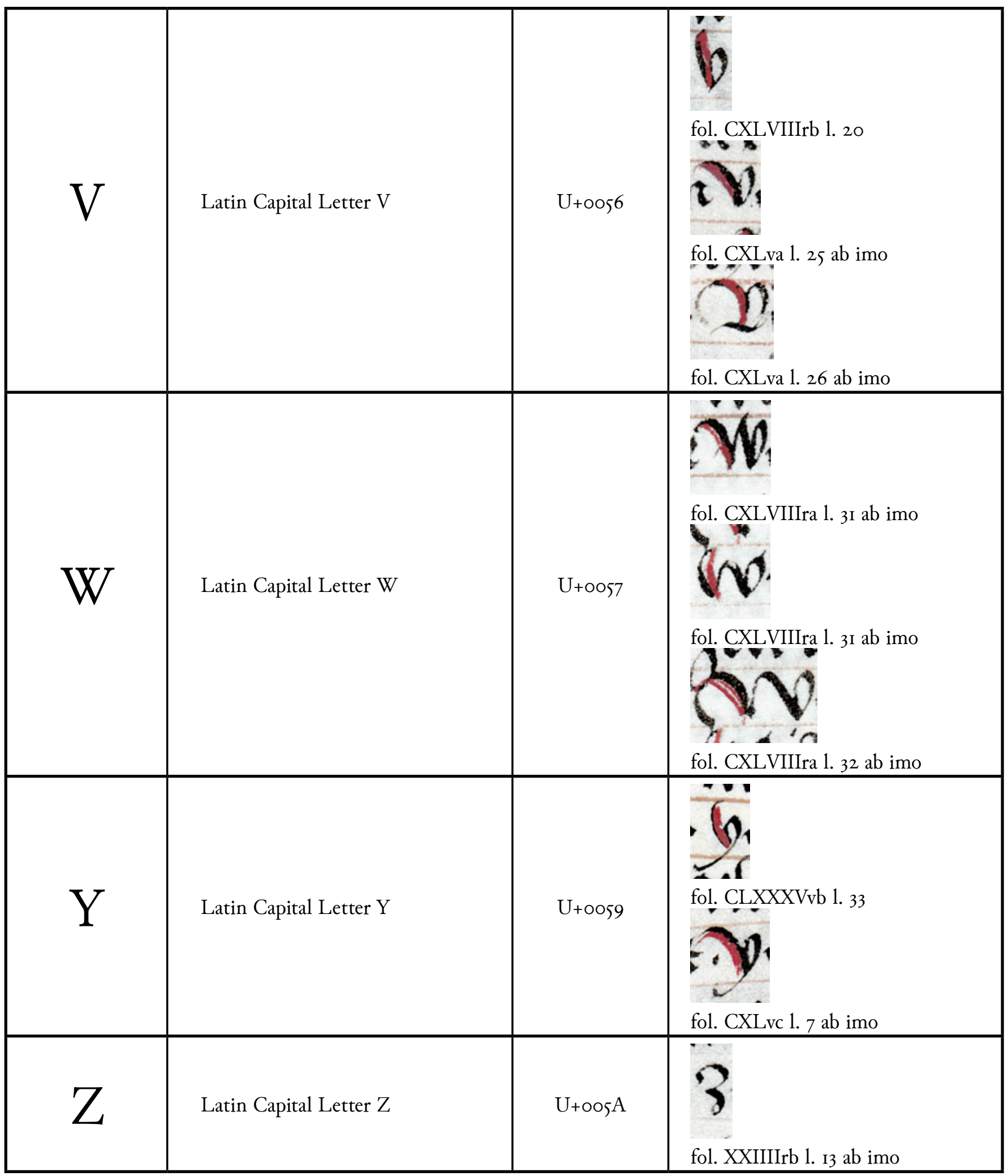




\section{Superskripta}

\begin{tabular}{|c|c|c|c|}
\hline $\begin{array}{l}\text { Transkriptions- } \\
\text { zeichen }\end{array}$ & Name & Unicode & Abbildung \\
\hline$u$ & $\begin{array}{l}\text { Combining Latin Small Letter } \\
\text { Flattened Open A Above }\end{array}$ & $\mathrm{U}_{+\mathrm{I}} \mathrm{DD}_{3}$ & fol. XCVrc l. 13 \\
\hline $\mathbf{O}$ & Combining Latin Small Letter O & $\mathrm{U}+0366$ & fol. XCVvb $1.6 \mathrm{ab} \mathrm{imo}$ \\
\hline$\smile$ & Combining Breve & $\mathrm{U}+0306$ & fol. XCVrc 1.6 \\
\hline
\end{tabular}




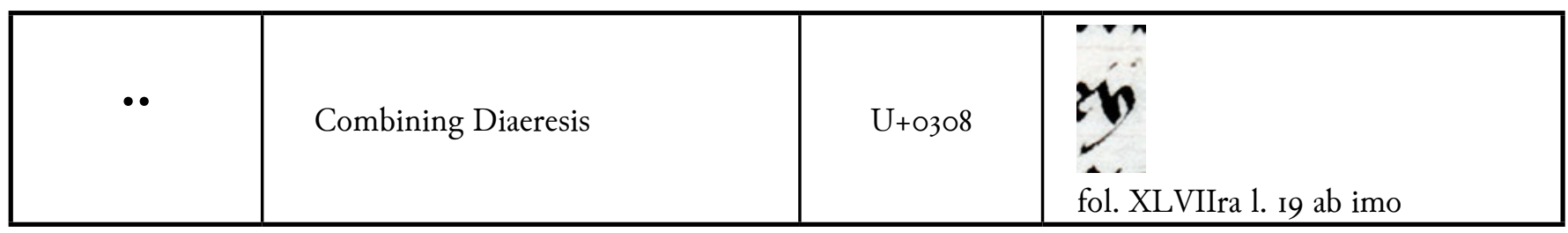

\section{Abkürzungszeichen}

\begin{tabular}{|c|c|c|c|}
\hline $\begin{array}{l}\text { Transkriptions- } \\
\text { zeichen }\end{array}$ & Name & Unicode & Abbildung \\
\hline- & Combining Macron & $\mathrm{U}+0304$ & fol. XLVva 1. I2 \\
\hline ? & Modifier Letter Glottal Stop & $\mathrm{U}+\mathrm{O}_{2} \mathrm{Co}$ & fol. CXCVIIrb $1.22 \mathrm{ab} \mathrm{imo}$ \\
\hline
\end{tabular}




\section{Interpunktionszeichen}

\begin{tabular}{|c|c|c|c|}
\hline $\begin{array}{c}\text { Transkriptions- } \\
\text { zeichen }\end{array}$ & Name & Unicode & Abbildung \\
\hline & & & \\
& Middle Dot & U+ooB7 & \\
& & & fol. XLVvb 1. I3 \\
\hline
\end{tabular}

Der Punkt markiert das Ende eines Verses. Vor dem Punkt und dahinter, falls danach noch weiterer Text folgt, wird jeweils ein Leerzeichen gesetzt.

\begin{tabular}{|c|c|c|c|}
\hline$/$ & Short Virgula & U+FIF7 &, \\
\hline
\end{tabular}

Der Schrägstrich markiert eine Zäsur innerhalb eines Verses oder das Ende eines Verses. Vor dem Schrägstrich und dahinter, falls danach noch weiter Text folgt, wird jeweils ein Leerzeichen gesetzt.

\begin{tabular}{|l|l|l|l|}
\hline & & & \\
& Colon & U+oo3A & $\vdots$ \\
& & & fol. XLVra 1. I5 ab imo \\
\hline
\end{tabular}

Der Doppelpunkt markiert das Ende eines Verses. Vor dem Doppelpunkt und dahinter, falls danach noch weiterer Text folgt, wird jeweils ein Leerzeichen gesetzt.

\begin{tabular}{|c|c|c|c|}
\hline$\sim$ & Tilde & $\mathrm{U}+007 \mathrm{E}$ & $\begin{array}{l}0 \\
\text { fol. Iva } 1.7\end{array}$ \\
\hline
\end{tabular}

Die Tilde markiert das Ende eines Verses. Vor der Tilde und dahinter, falls danach noch weiterer Text folgt, wird jeweils ein Leerzeichen gesetzt.

\begin{tabular}{|c|c|c|c|}
\hline$=$ & Double Oblique Hyphen & $\mathrm{U}+2 \mathrm{E} 17$ & $\begin{array}{l}\text { l, } \\
\text { fol. CXVIIrc 1. I6 }\end{array}$ \\
\hline \multicolumn{4}{|c|}{$\begin{array}{l}\text { Der Doppelbindestrich markiert eine Worttrennung, bei der das Wort in der nächsten Zeile fortgeführt wird. Vor } \\
\text { dem Doppelbindestrich wird kein Leerzeichen gesetzt. }\end{array}$} \\
\hline$\Lambda$ & Logical And & $U+2227$ & $\begin{array}{l}\text { fol. XXIIvb } 1.34 \\
\text { fis }\end{array}$ \\
\hline
\end{tabular}




\subsection{Diplomatische Transkription (rechte Seite der Gesamtausgabe, rechte Spalte)}

Auf der rechten Seite der Gesamtausgabe befindet sich die diplomatische Transkription, die alle allographischen Varianten zu den entsprechenden Graphemen (siehe Auflistung der Kleinbuchstaben) vereinheitlicht. Alle $\mathrm{Ab}$ breviaturen werden aufgelöst sowie ausgepunktete und durchgestrichene Textpassagen werden getilgt. Ebenso wird auf die Interpunktionszeichen in der diplomatischen Transkription verzichtet. Lombarden sowie Incipits und Explicits werden wie in der allographischen Transkription farblich (rot oder blau) hervorgehoben. Initialen werden mittels Fettdruck hervorgehoben. Die Superskripta werden gemäß den folgenden Regeln transformiert, um die allgemeine Zitierbarkeit der diplomatischen Transkription zu gewährleisten.

\section{Transformationen von Superskripta}

\section{Superskriptum a:}

Beim Superskriptum a, das in der allographischen Transkription ein eigenes Zeichen $\left(\mathrm{U}_{\mathrm{I}} \mathrm{ID}_{3}\right)$ darstellt, muss unterschieden werden, über welchem Buchstaben es sich befindet. Es ergibt sich daraus eine Bedeutungsunterscheidung, die bei der Transformation des Zeichens beachtet werden muss. Im Folgenden werden die verschiedenen Buchstaben, über denen sich das Superskriptum a in der Transkription befinden kann, aufgelistet und gezeigt, wie die Zeichen transformiert werden.

- Das Superskriptum a über «y wird getilgt, da es keine bedeutungstragende Funktion hat, sondern rein graphischer Natur ist.

$$
\text { Beispiel: wey̆b } \rightarrow \text { weyb }
$$

- Die Kombination aus 〈a und Superskriptum a wird zum Umlaut 〈ä〉 transformiert.

Beispiel: măre $\rightarrow$ märe

- Das Superskriptum a über «e` wird getilgt, da es keine bedeutungstragende Funktion hat, sondern rein graphischer Natur ist.

Beispiel: wëre $\rightarrow$ were

- Die Kombination aus $\langle 0\rangle$ und Superskriptum a wird zum Umlaut 〈ö transformiert.

Beispiel: möcht $\rightarrow$ möcht

- Bei der Kombination aus «u und Superskriptum a muss unterschieden werden, ob sich das $<\mathrm{u}>$ in der Schreibung eines Diphthongs, dargestellt durch 〈e〉 und 〈u〉, befindet oder ob das Superskriptum a über «u einen Umlaut markiert.
- Die Kombination aus « $\mathrm{u}$ und Superskriptum a wird zum Umlaut «̈̈ transformiert.

Beispiel: Fürften $\rightarrow$ Fürsten

- Befindet sich das Superskriptum a über $\langle\mathrm{u}\rangle$ in der Schreibung eines Diphthongs, dargestellt durch 〈e〉 und $\langle\mathrm{u}$, wird das Superskriptum a getilgt, da es keine bedeutungstragende Funktion hat, sondern rein graphischer Natur ist.

Beispiel: leüten $\rightarrow$ leuten

- Die Kombination aus 〈v〉 und Superskriptum a wird zur Kombination aus $\langle\mathrm{v}\rangle$ und Trema transformiert.

Beispiel: v้ntz $\rightarrow$ v̈ntz

- Das Superskriptum a über 〈w〉 wird getilgt, da es keine bedeutungstragende Funktion hat, sondern rein graphischer Natur ist.

Beispiel: frew $\stackrel{u}{\mathrm{e}} \rightarrow$ frewen

Superskriptum o:

Das Superskriptum o, das in der allographischen Transkription ein eigenes Zeichen $(U+0366)$ darstellt, wird durch «o〉 ersetzt. Dabei kommt es zu keiner Differenzierung, da das Superskriptum o stets über $<\mathrm{u}$ oder vokalischem 〈v〉 vorkommt und einen Diphthong markiert.

Beispiel: gůt $\rightarrow$ guot; $\stackrel{\circ}{\text { Vte }} \rightarrow$ Vote

Breve:

Das Breve, das in der allographischen Transkription ein eigenes Zeichen (U+0306) darstellt, wird ersatzlos gestrichen, da ihm eine rein graphische Funktion zur Kenntlichmachung von Vokalen (〈u〉, 〈W〉) in Abgrenzung zu Konsonanten (wie z. B. 〈n〉) zukommt oder es als Dehnungszeichen über Vokalen (〈a, $\langle$ ২) verwendet wird, wobei diese Fälle vernachlässigbar sind.

Beispiel: paŭm $\rightarrow$ paum; trew $\rightarrow$ trew; ăn $\rightarrow$ an; ŏne $\rightarrow$ one

\section{Trema:}

Beim Trema, das in der allographischen Transkription ein eigenes Zeichen $(U+0308)$ darstellt, muss unterschieden werden, über welchem Buchstaben es sich befindet. Es ergibt sich daraus eine Bedeutungsunterscheidung, die bei der Transformation des Zeichens beachtet werden muss. Im Folgenden werden die verschiedenen Buchstaben, über denen sich das Trema in der Transkription befinden kann, aufgelistet und gezeigt, wie die Zeichen transformiert werden.

- Das Trema über 〈y〉 wird getilgt, da es keine bedeutungstragende Funktion hat, sondern rein graphischer Natur ist. 
Beispiel: nÿemand $\rightarrow$ nyemand

- Die Kombination aus «a und Trema wird zum Umlaut (ä) transformiert.

Beispiel: märe $\rightarrow$ märe

- Die Kombination aus $\langle 0\rangle$ und Trema wird zum Umlaut 〈Ö〉 transformiert.

Beispiel: möcht $\rightarrow$ möcht

- Die Kombination aus «u und Trema wird zum Umlaut «ü transformiert.

Beispiel: künig $\rightarrow$ künig

- Die Kombination aus 〈 $\mathrm{v}\rangle$ und Trema bleibt unverändert, da es für diese Kombination kein eigenständiges Zeichen wie für $\langle\ddot{a}\rangle,\langle\ddot{o}\rangle$ oder $\langle\ddot{\mathrm{u}}\rangle$ gibt.

- Das Trema über «e wird getilgt, da es keine bedeutungstragende Funktion hat, sondern rein graphischer Natur ist.

Beispiel: wëne $\rightarrow$ wene

\section{Vers- und Strophengliederung}

Im Gegensatz zur Zeilengliederung des Manuskripts, die in der allographischen Transkription beibehalten wird, bedient sich die diplomatische Transkription einer Vers- und Strophengliederung, die stets die vollständigen Verse aus der allographischen Transkription wiedergibt. Die Verse und Strophen sind gemäß etablierten Editionen nummeriert, um die Vergleichbarkeit mit diesen Editionen zu ermöglichen. Zusatzverse und -strophen sind mit Kleinbuchstaben nach den Nummern versehen. Das >Nibelungenlied im >Ambraser Heldenbuch ‘ folgt bei der Nummerierung der Strophen Handschrift B. Bei Strophen, die sich nicht in Handschrift B finden, sind die Siglen der Handschriften A und C oder, falls diese sich dort auch nicht finden, die Siglen der Handschriften a und $\mathrm{k}$ vor den Strophennummern angegeben. Es wird jedoch in allen Fällen die Abfolge der Verse und Strophen, wie sie im >Ambraser Heldenbuch vorliegt, beibehalten und nicht der Reihenfolge der Verse und Strophen in den etablierten Editionen angepasst. Bei jeder Abweichung der Reihenfolge der Verse gegenüber den etablierten Editionen werden die jeweils betroffenen Verse stets nummeriert. Damit sind jegliche Diskrepanzen der Versabfolge im >Ambraser Heldenbuch gegenüber den etablierten Editionen auf den ersten Blick ersichtlich. Ansonsten wird nur jeder fünfte Vers nummeriert.
Hierbei wird in der diplomatischen Transkription immer der Vers- und Strophenbestand des >Ambraser Heldenbuchs` ungeachtet von Reiminkonsistenzen möglichst ohne editorische Eingriffe abgebildet. Bei fehlenden oder fehlerhaften Markierungen für Versgrenzen oder bei ausgefallenen >Reimwörtern` wurden Versumbrüche nach Plausibilität oder gemäß etablierten Editionen vorgenommen.

Neben den Incipits der Texte mit âventiuren (>Nibelungenlied`, >Kudrun`, >Biterolf und Dietleib`, >Ortnit‘, ,Wolfdietrich A $)$ befinden sich die Nummern der jeweiligen âventiuren. Wenn das Incipit nicht vorhanden ist, sich an einer falschen Stelle befindet oder sich im Incipit nicht das Wort "Abenteuer « befindet, ist ein durch eckige Klammern eingefasster Zusatz hinzugefügt, der angibt, um welche âventiure es sich handelt.

Bei Werken mit Langzeilen (>Nibelungenlied`, >Ku-

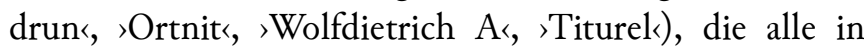
Strophen gegliedert sind, befinden sich die Incipits, Explicits sowie die Strophenanfänge in der diplomatischen Transkription auf gleicher Höhe mit den jeweiligen Incipits, Explicits sowie den Strophenanfängen in der allographischen Transkription. Aus satztechnischen Gründen musste in einigen wenigen Ausnahmefällen auf eine synoptische Anordnung verzichtet werden, wobei es zu leichten Abweichungen von ein bis zwei Verszeilen der Strophenanfänge gegenüber der allographischen Transkription kommen kann. Da Strophenanfänge in der Regel vom Schreiber durch abwechselnd rote oder blaue Lombarden gekennzeichnet wurden, die sowohl in der allographischen als auch der diplomatischen Transkription in der jeweiligen Farbe wiedergegeben sind, ist auch in diesen Fällen eine leichte Orientierung sichergestellt. Bei der >Rabenschlacht - dem einzigen Text mit Strophengliederung ohne Langzeilen - musste aus Platzgründen wie bei allen anderen Texten ohne Langzeilen auf eine synoptische Gegenüberstellung von allographischer und diplomatischer Transkription gänzlich verzichtet werden. Auch hier dienen wiederum die farbigen Lombarden als Orientierungshilfe. Lediglich auf der jeweils ersten Seite aller Texte (mit Ausnahme von >Die Frauenehre Craûn $\varsigma$ findet sich eine synoptische Gegenüberstellung der Incipits und ersten Verse. Bei >Biterolf und Dietleib sind darüber hinaus alle Incipits synoptisch angeordnet. 


\section{Bibliographie}

\section{I. Konsultierte Editionen für Nummerierung der Verse und Strophen}

Die Nummerierung der Verse und Strophen der Werke des >Ambraser Heldenbuchs in der vorliegenden Gesamttranskription basiert auf folgenden Editionen:

Der Stricker: >Die Frauenehre`:

Hofmann, Klaus (Hrsg.) (1976): Strickers >Frauenebres: Überlieferung, Textkritik, Edition, literaturgescbicbtliche Einordnung. Marburg: N. G. Elwert, S. II7-I55.

>Mauritius von Craûn`:

Reinitzer, Heimo (Hrsg.) (200o): Mauritius von Craûn. Altdeutsche Textbibliothek Nr. II3. Tübingen: Niemeyer, S. I-93.

Hartmann von Aue: >Iwein`:

Hartmann von Aue (200I): Iwein. 4., überarbeitete Auflage. Text der siebenten Ausgabe von G. F. Benecke, K. Lachmann und L. Wolff. Übersetzung und Nachwort von Thomas Cramer. Berlin: De Gruyter, S. 3-I47.

Hartmann von Aue: >Die Klage`:

Hartmann von Aue (2015): Die Klage. Herausgegeben von Kurt Gärtner. Altdeutsche Textbibliothek Nr. I23. Berlin: De Gruyter, S. I-80.

>Das Büchlein`:

Hartmann von Aue (1972): Das Klagebüchlein Hartmanns von Aue und Das zweite Büchlein. Herausgegeben von Ludwig Wolff. Altdeutsche Texte in kritischen Ausgaben Band 4. München: Wilhelm Fink, S. 7I-9I.

>Der Mantel:

Schröder, Werner (Hrsg.) (1995): Das Ambraser MantelFragment. Nach der einzigen Handschrift neu herausgegeben von Werner Schröder. Sitzungsberichte der Wissenschaftlichen Gesellschaft an der Johann Wolfgang Goethe-Universität Frankfurt am Main Band XXXIII Nr. 5. Stuttgart: Franz Steiner, S. 132-165.
Hartmann von Aue (2017): Ereck: Textgeschichtliche Ausgabe mit Abdruck sämtlicher Fragmente und der Bruchstücke des mitteldeutschen , Erekr. Herausgegeben von Andreas Hammer, Victor Millet und Timo Reuvekamp-Felber unter Mitarbeit von Lydia Merten, Katharina Münstermann und Hannah Rieger. Berlin: De Gruyter, S. 2-52.

Hartmann von Aue: ১Erec $`$

Hartmann von Aue (2006): Erec: Mit einem Abdruck der neuen Wolfenbütteler und Zwettler Erec-Fragmente. Herausgegeben von Albert Leitzmann, fortgeführt von Ludwig Wolff. 7. Auflage besorgt von Kurt Gärtner. Altdeutsche Textbibliothek Nr. 39. Tübingen: Niemeyer, S. I-297.

>Dietrichs Flucht $<$ :

Lienert, Elisabeth/Beck, Gertrud (Hrsg.) (2003): Dietrichs Flucht: Textgeschichtliche Ausgabe. Texte und Studien zur mittelhochdeutschen Heldenepik Band I. Tübingen: Niemeyer, S. 3-299.

>Rabenschlacht<:

Lienert, Elisabeth/Wolter, Dorit (Hrsg.) (2005): Rabenschlacht: Textgeschichtliche Ausgabe. Texte und Studien zur mittelhochdeutschen Heldenepik Band 2. Tübingen: Niemeyer, S. 3-235.

>Nibelungenlied

Batts, Michael S. (Hrsg.) (197I): Das Nibelungenlied: Paralleldruck der Handschriften A, B und C nebst Lesarten der übrigen Handschriften. Tübingen: Niemeyer, S. 2-65I.

>Nibelungenklage`:

Bumke, Joachim (Hrsg.) (1999): Die sNibelungenklager: Synoptische Ausgabe aller vier Fassungen. Berlin: De Gruyter, S. 40-494.

`Kudrun`:

Stackmann, Karl (Hrsg.) (200o): Kudrun. Nach der Ausgabe von Karl Bartsch herausgegeben von Karl Stackmann. Altdeutsche Textbibliothek Nr. II5. Tübingen: Niemeyer, S. I-337. 
$>$ Biterolf und Dietleib<:

Jänicke, Oskar (Hrsg.) (ı866): Biterolf und Dietleib. Herausgegeben von Oskar Jänicke. Laurin und Walberan. Mit Benutzung der von Franz Roth gesammelten Abschriften und Vergleichungen. Deutsches Heldenbuch Erster Teil. Berlin: Weidmannsche Buchhandlung, S. I-I97.

Schnyder, André (Hrsg.) (1980): Biterolf und Dietleib. Neu herausgegeben und eingeleitet von André Schnyder. Sprache und Dichtung Neue Folge Band 3r. Bern: Paul Haupt, S. 79-417.

〉ortnit<:

Kofler, Walter (Hrsg.) (2009): Ortnit und Wolfdietrich A. Stuttgart: Hirzel, S. 29-I03.

,Wolfdietrich A<:

Kofler, Walter (Hrsg.) (2009): Ortnit und Wolfdietrich A. Stuttgart: Hirzel, S. I07-167.

>Die böse Frau<:

Ebbinghaus, Ernst A. (Hrsg.) (1968): Daz buoch von dem übeln wîbe. 2., neubearbeitete Auflage. Altdeutsche Textbibliothek Nr. 46. Tübingen: Niemeyer, S. I-33.

Herrand von Wildonie: >Die treue Gattin`:

Herrand von Wildonie (1969): Vier Erzäblungen. Herausgegeben von Hanns Fischer. Zweite, revidierte Auflage besorgt von Paul Sappler. Altdeutsche Textbibliothek Nr. 5I. Tübingen: Niemeyer, S. I-9.

Herrand von Wildonie: >Der betrogene Gatte:

Herrand von Wildonie (1969): Vier Erzäblungen. Herausgegeben von Hanns Fischer. Zweite, revidierte Auflage besorgt von Paul Sappler. Altdeutsche Textbibliothek Nr. 5I. Tübingen: Niemeyer, S. IO-2I.

Herrand von Wildonie: >Der nackte Kaiser $<$ :

Herrand von Wildonie (1969): Vier Erzäblungen. Herausgegeben von Hanns Fischer. Zweite, revidierte Auflage besorgt von Paul Sappler. Altdeutsche Textbibliothek Nr. 5I. Tübingen: Niemeyer, S. 22-43.
Herrand von Wildonie: >Die Katze`:

Herrand von Wildonie (1969): Vier Erzählungen. Herausgegeben von Hanns Fischer. Zweite, revidierte Auflage besorgt von Paul Sappler. Altdeutsche Textbibliothek Nr. 5I. Tübingen: Niemeyer, S. 44-53.

Ulrich von Liechtenstein: >Frauenbuch`:

Ulrich von Liechtenstein (1993): Frauenbuch. 2., durchgesehene Auflage. Herausgegeben von Franz Viktor Spechtler. Göppinger Arbeiten zur Germanistik Nr. 520. Göppingen: Kümmerle, S. 2-I22.

Wernher der Gärtner: `Helmbrecht`:

Wernher der Gartenære (1993): Helmbrecht. Herausgegeben von Friedrich Panzer und Kurt Ruh. Io. Auflage besorgt von Hans-Joachim Ziegeler. Altdeutsche Textbibliothek Nr. II. Tübingen: Niemeyer, S. I-77.

Der Stricker: >Pfaffe Amis`:

Henne, Hermann (Hrsg.) (I99I): Der Pfaffe Amis von dem Stricker: Ein Schwankroman aus dem I3. Jabrhundert in zwölf Episoden. Herausgegeben und übersetzt von Hermann Henne. Göppinger Arbeiten zur Germanistik Nr. 530. Göppingen: Kümmerle, S. 9-108.

Wolfram von Eschenbach: >Titurek:

Wolfram von Eschenbach (2006): Titurel: Mit der gesamten Parallelüberlieferung des »Jüngeren Titurel«. Kritisch herausgegeben, übersetzt und kommentiert von Joachim Bumke und Joachim Heinzle. Tübingen: Niemeyer, S. 2-44.

>Brief des Priesterkönigs Johannes`:

Zarncke, Friedrich (Hrsg.) (I879): »Der Priester Johannes: Erste Abhandlung: Enthaltend Capitel I, II und III", in: Abbandlungen der philologisch-bistorischen Classe der Königlich Sächsischen Gesellschaft der Wissenschaften Siebenter Band. Leipzig: Hirzel, S. 827-I030, hier: S. $957-968$.

Roth, F. W. E. (Hrsg.) (I895): »Von dem reichtumb priester Johanns«, in: Zeitschrift für deutsche Pbilologie 27, S. 216-248, hier: S. 219-245. 


\subsection{Zitierte Sekundärliteratur}

Im Folgenden ist die Literatur aufgelistet, die in der Einleitung der vorliegenden Gesamttranskription zitiert wurde. Für einen umfassenden Überblick über die Forschungsliteratur zum >Ambraser Heldenbuch siehe Janota (1978), Gärtner (2015) und Klarer (2019).

Alisade, Hubert (2019): »Zur Entstehungsgeschichte des Ambraser Heldenbuchs: Die Beauftragung Hans Rieds«, in: Klarer, Mario (Hrsg.): Kaiser Maximilian I. und das Ambraser Heldenbuch. Wien: Böhlau, S. 27-35.

Bäuml, Franz H. (I969a): »Das Ambraser Heldenbuch«, in: Kudrun: Die Handschrift. Herausgegeben von Franz H. Bäuml. Berlin: De Gruyter, S. I-I9.

Bäuml, Franz H. (1969b): »Die Kudrun Handschrift«, in: Kudrun: Die Handscbrift. Herausgegeben von Franz H. Bäuml. Berlin: De Gruyter, S. 20-45.

Domanski, Kristina (2019): „Zwischen Naturstudium und Dekor: Kunsthistorische Bemerkungen zum gemalten Buchschmuck im Ambraser Heldenbucb«, in: Klarer, Mario (Hrsg.): Kaiser Maximilian I. und das Ambraser Heldenbuch. Wien: Böhlau, S. 145-169.

Gärtner, Kurt (2006): Einleitung«, in: Hartmann von Aue: Erec: Mit einem Abdruck der neuen Wolfenbütteler und Zwettler Erec-Fragmente. Herausgegeben von Albert Leitzmann, fortgeführt von Ludwig Wolff. 7. Auflage besorgt von Kurt Gärtner. Altdeutsche Textbibliothek Nr. 39. Tübingen: Niemeyer, S. XI-XLIII.

Gärtner, Kurt (2015): »Einleitung«, in: Hartmann von Aue: Die Klage. Herausgegeben von Kurt Gärtner. Altdeutsche Textbibliothek Nr. I23. Berlin: De Gruyter, S. IX-XXXVIII.

Homeyer, Susanne/Knor, Inta (2015): »Zu einer umfassenden Untersuchung der Schreibsprache Hans Rieds im Ambraser Heldenbuch«, in: Zeitschrift für deutsche Philologie I34(I), S. 97-I03.

Janota, Johannes (1978): »Ambraser Heldenbuch«", in: Die deutsche Literatur des Mittelalters: Verfasserlexikon. Begründet von Wolfgang Stammler, fortgeführt von Karl Langosch. Zweite, völlig neu bearbeitete Auflage unter Mitarbeit zahlreicher Fachgelehrter herausgegeben von Kurt Ruh zusammen mit Gundolf Keil,
Werner Schröder, Burghart Wachinger, Franz Josef Worstbrock. Redaktion Kurt Illing, Christine Stöllinger. Band I $>$ A solis ortus cardines - Colmarer Dominikanerchronist. Berlin: De Gruyter, Sp. 323-327.

Klarer, Mario (Hrsg.) (2019): Kaiser Maximilian I. und das Ambraser Heldenbuch. Wien: Böhlau.

Leitzmann, Albert (1935): »Die Ambraser Erecüberlieferung«, in: Beiträge zur Geschicbte der deutschen Sprache und Literatur 59, S. 143-234.

Menhardt, Hermann (196I): Verzeichnis der altdeutschen literarischen Handscbriften der Österreichischen Nationalbibliothek. 3. Band. Veröffentlichungen des Instituts für deutsche Sprache und Literatur I3. Berlin: AkademieVerlag.

Mura, Angela (2007): „Spuren einer verlorenen Bibliothek: Bozen und seine Rolle bei der Entstehung des Ambraser Heldenbuchs ( $1504-1516) \ll$, in: cristallin wort: Hartmann-Studien I, S. 59-128.

Tratter, Aaron (2019): ßBuchschmuck, Lagen, leere Seiten: Was kodikologische Merkmale über den Entstehungsprozess des Ambraser Heldenbucbs verraten können«, in: Klarer, Mario (Hrsg.): Kaiser Maximilian I. und das Ambraser Heldenbuch. Wien: Böhlau, S. 37-48.

Unterkircher, Franz (1973): Ambraser Heldenbuch: Kommentar. Codices Selecti Vol. XLIII. Graz: Akademische Druck- u. Verlagsanstalt.

\subsection{Faksimile}

Ambraser Heldenbuch: Vollständige Faksimile-Ausgabe im Originalformat des Codex Vindobonensis series nova 2663 der Österreichischen Nationalbibliothek. Codices Selecti Vol. XLIII. Graz: Akademische Druck- u. Verlagsanstalt. 1973.

\section{Abbildungen}

Alle Abbildungen in diesem Band stammen aus >Ambraser Heldenbuch`, Wien, Österreichische Nationalbibliothek, Cod. ser. nova 2663. 